\title{
Synthesis of Diazepam-Imprinted Polymers with Two Functional Monomers in Chloroform Using a Bulk Polymerization Method
}

\author{
Aliya Nur Hasanah $\left(\mathbb{D},{ }^{1}\right.$ Dang Soni, ${ }^{1,2}$ Rimadani Pratiwi $\mathbb{D}^{1},{ }^{1}$ Driyanti Rahayu, \\ Sandra Megantara, ${ }^{1}$ and Mutakin ${ }^{1}$ \\ ${ }^{1}$ Department of Pharmaceutical Analysis and Medicinal Chemistry, Faculty of Pharmacy, Padjadjaran University, \\ Jl. Raya Bandung Sumedang KM 21,5 Jatinangor, Sumedang, Indonesia \\ ${ }^{2}$ Pharmacy Department, Universitas Garut, Garut, West Java, Indonesia \\ Correspondence should be addressed to Aliya Nur Hasanah; aliya.n.hasanah@unpad.ac.id
}

Received 23 August 2019; Revised 13 December 2019; Accepted 27 December 2019; Published 18 February 2020

Academic Editor: Hassan Arida

Copyright (c) 2020 Aliya Nur Hasanah et al. This is an open access article distributed under the Creative Commons Attribution License, which permits unrestricted use, distribution, and reproduction in any medium, provided the original work is properly cited.

\begin{abstract}
Diazepam is a benzodiazepine that has the potency to be misused because it is effective, easily obtained, and inexpensive. The misuse of diazepam is to replace illegal drugs and be a sedative. Separation of diazepam is needed to detect possible drug abuse and to monitor drug levels in blood to ensure the effectiveness of the drug. This study was conducted to obtain a molecularly imprinted solid-phase extraction (MI-SPE) sorbent to separate diazepam from serum samples. This work started at the synthesis stage with the bulk polymerization method, using methyl methacrylate and acrylamide as functional monomers, diazepam as a template, and ethylene glycol dimethacrylate as a crosslinker. The polymer obtained was identified by its adsorption capacity and packaged into a solid-phase extraction (SPE) cartridge, and the extraction conditions were optimized. The optimization results were then used to extract diazepam from the serum sample. The test results showed that the adsorption ability of the molecularly imprinted polymer (MIP) with the functional monomer, methyl methacrylate, was $63.98 \pm 0.1 \%$, which is higher than that of the acrylamide MIP monomer, with a value of $43.27 \pm 0.1 \%$. The MIP sorbent of methyl methacrylate was applied to the SPE with $200 \mathrm{mg}$ of polymer in a $3 \mathrm{~mL}$ cartridge. Diazepam added to serum samples were then passed through the MIP-SPE producing a percent recovery value of $95.31 \pm 1.1 \%$ for MIP and $60.83 \pm 0.3 \%$ for nonimprinted polymer (NIP). The results showed that the MI-SPE sorbent made from the monomer methyl methacrylate gave higher extraction recovery results than acrylamide, and it could be used for extracting diazepam from serum samples with or without other substances.
\end{abstract}

\section{Introduction}

Diazepam is a drug from the benzodiazepine class that can affect the central nervous system and provide a sedative effect by influencing neurotransmitters that function to transmit signals to brain cells. Diazepam is commonly used to treat acute alcohol withdrawal symptoms, anxiety disorders, insomnia, and convulsions. Diazepam is a benzodiazepine that is often prescribed and misused in several countries because it is cheap, effective, and easily obtained [1]. Diazepam drug abuse can cause addiction. According to the Drug Enforcement Administration (DEA), more than 20 million people abuse benzodiazepine, including diazepam
[2]. This abuse of benzodiazepine is often done to replace illegal drugs, such as opiates, tranquilizers, and neuroleptics [3]. Benzodiazepine drugs can also contribute to sudden death if misused, and more than 7,900 people die from overdoses involving diazepam each year. Drug analysis is fundamental on drug therapy monitoring and identifying drug abuse easily [4].

In general, diazepam can be analysed qualitatively by thin-layer chromatography [5] and analysed quantitatively by ultraviolet spectrometry or high-performance liquid chromatography (HPLC) [6], but the method is less selective and less sensitive if used to quantify samples with small and complex amounts of analytes, such as biological fluids. 
Therefore, for liquid biological samples, high-performance liquid chromatography-tandem mass spectrometry [7] or gas chromatography-tandem mass chromatography $[8,9]$ is usually used because of their high selectivity. An option to increase the sensitivity of the analytical method is through selective sample preparation.

Molecularly imprinted solid-phase extraction (MI-SPE) is the combination of molecularly imprinted polymer (MIP) and solid-phase extraction (SPE) methods, and it is becoming increasingly popular for selective extraction of various analytes from complex matrices, such as serum and urine. A lot of research studies develop MIP for extraction and analysis of analytes from complex matrices such as metaproterenol [10], clenbuterol [11], atenolol [12], oxazepam [13], and triamterene [14]. An MIP was first made by Wulff and Sarhan through the synthesis of polymers that use target analytes as a template, so that after elution, a cavity will be formed, which will be able to selectively bind the analytes in the sample [15]. The commonly used polymerization technique in the process of making an MIP is bulk polymerization due to its simplicity and lack of expertise requirement [16].

Another study made an MIP for diazepam using methacrylic acid as a functional monomer, with a recovery percentage of $88 \%$ in the serum and urine sample [17]. This recovery is still low and needs enhancement. So far, studies on diazepam MIP used methacrylic acid as a functional monomer $[3,17]$. According to that result, in this study, an MIP was made using bulk polymerization techniques with acrylamide (AAM) and methyl methacrylate (MMA) as functional monomers, prepared separately. The selection of these monomers is based on the properties of diazepam as a weak acid. In this work, polymers were made using chloroform as a porogenic solvent, and use of the polymers as SPE materials for diazepam from serum samples was evaluated.

\section{Materials and Methods}

2.1. Materials. Diazepam (DZM) was purchased from Merck. AAM was from Sigma-Aldrich. Acetonitrile (ACN) and methanol $(\mathrm{MeOH})$ were purchased from Fisher Chemical. Glibenclamide (GLIB) and gliclazide (GLIC) were purchased from Sigma-Aldrich. Acetic acid (AcOH) (96\%), chloroform, ethanol, benzoyl peroxide (BPO), and potassium bromide were purchased from Merck. Ethylene glycol dimethacrylate (EGDMA) and MMA were purchased from Sigma-Aldrich. All chemicals used were commercially available and were of analytical grade. Methanol used was of HPLC gradient grade. Methyl methacrylate was purified before being used with aluminium chloride.

\subsection{Computational Approach on Prediction of Monomer-} Template Interaction. Evaluations of the interactions and hydrogen bonding between the template and monomers were carried out using a computational approach. Twodimensional structures of DZM and monomers chosen (AAM and MMA) were drawn and then converted into three dimensions using the ChemBio3D Ultra 12.0 program. Geometry optimization was then carried out with the $a b$ initio method (Hartree-Fock, base set 3-21G) using the games interface on ChemBio3D Ultra 12.0. The docking process between DZM and AAM/MMA was undertaken using the PyRx software virtual screening tools with AutoDock Vina. The docking results were analysed by comparing the position and type of bond formed and the value of the binding affinity of the template and each monomer using the AutoDockTools 1.5 software.

2.3. Determination of the Association Constant of the Monomer-Template. DZM $\left(1 \times 10^{-5} \mathrm{M}\right)$ was diluted in chloroform. Two $\mathrm{mL}$ of the solution was measured in a UVVis spectrophotometer. The MMA/AAM solution (0.01 M) was added gradually from $10 \mu \mathrm{L}, 60 \mu \mathrm{L}, 110 \mu \mathrm{L}, 210 \mu \mathrm{L}$, $260 \mu \mathrm{L}, 310 \mu \mathrm{L}, 360 \mu \mathrm{L}$, and so on until there was no significant increase in the absorbance value. The results were plotted as a graph of $1 /[G]$ vs. $1 / \Delta Y$. The association constant was calculated using the Benesi-Hildebrand equation [18] as follows:

$$
\frac{1}{\Delta Y}=\frac{1}{Y \Delta H G K a[G]}+\frac{1}{Y \Delta H G},
$$

$\Delta Y$ is the change in absorbance, $Y \Delta H G$ is the change in absorbance at the end of the titration, and $[G]$ is the concentration of the monomer added [19].

\subsection{Synthesis of the Diazepam-Imprinted Polymer Using Bulk} Polymerization. A prepolymerization solution consisting of $0.263 \mathrm{~g}$ diazepam, $400 \mu \mathrm{L}$ MMA, $3.77 \mathrm{~mL}$ EGDMA, $250 \mathrm{mg}$ $\mathrm{BPO}$, and $5 \mathrm{~mL}$ chloroform was prepared in a vial for DZMMIP (diazepam molecular-imprinted polymer) of MMA. $0.263 \mathrm{~g}$ diazepam, $284.32 \mathrm{mg}$ AAM, $3.77 \mathrm{~mL}$ EGDMA, $250 \mathrm{mg}$ BPO, and $5 \mathrm{~mL}$ chloroform were prepared in a vial for DZM-MIP (diazepam molecular-imprinted polymer) of AAM. The reaction molar ratio of each template, functional monomer, and crosslinker for the preparation of each MIP was $1: 4: 20$. Each solution was sonicated for 20 minutes to remove oxygen. The DZM-MIP was placed in an oven at $70^{\circ} \mathrm{C}$ for 1 hour, moved to a water bath, and kept at $70^{\circ} \mathrm{C}$ for 18 hours for polymerization. The resulting bulk polymers were ground and sieved using mesh 60, washed with $20 \mathrm{~mL}$ methanol, and dried at $50^{\circ} \mathrm{C}$. The nonimprinted polymer (NIP) was prepared simultaneously under the same conditions without the addition of a template [20-23]. A soxhlet apparatus was used for template removal from the synthesized MIP (molecular-imprinted polymer) using methanol and acetic acid $(9: 1)$ for 24 hours 3 times, each time with $200 \mathrm{~mL}$. Then, polymers were washed using $20 \mathrm{~mL}$ of methanol and water and dried at $50^{\circ} \mathrm{C}$ for 18 hours. The MIP was monitored using $20 \mathrm{mg}$ of the MIP diluted in $5 \mathrm{~mL}$ of chloroform, performed in triplicate. Then, the MIP was sonicated for 5 minutes and set aside for 24 hours. The extraction process was complete when MIP no longer contained the template when monitored using UV-Vis spectrophotometry [21]. 
2.5. Evaluation of Binding Ability. Twenty $\mathrm{mg}$ of the MIP in a vial was incorporated into $5 \mathrm{~mL}$ of DZM at a concentration of $5 \mathrm{mg} / \mathrm{L}$ in various solutions, such as ethanol, chloroform, and methanol, performed in triplicate. The vials were shaken using a shaker at $120 \mathrm{rpm}$ for 10 minutes and set aside for 18 hours at room temperature to reach equilibrium. They were then filtered. The filtrate was measured using UV-Vis spectrophotometry. The evaluation of the NIP was carried out by the same procedure as the MIP [21].

2.6. Evaluation of Binding Capacity. Five $\mathrm{mL}$ of the selected solvent determined from the evaluation conducted in Section 2.5 , containing 2, 4, 6, 8, and $10 \mathrm{mg} / \mathrm{L}$ of DZM were added to $20 \mathrm{mg}$ of the MIP in a vial, performed in triplicate. The vials were shaken using a shaker at $120 \mathrm{rpm}$ for 10 minutes at room temperature and set aside for 18 hours without shaking. They were then filtered. The filtrate was measured using UV-Vis spectrophotometry. The results were plotted to the isotherm Freundlich adsorption curve. The adsorption curve of the NIP was determined according to the same procedure as the MIP $[21,22]$.

2.7. Solid-Phase Extraction Optimization Condition. Two hundred mg of the MIP and the NIP from AAM and MMA were packed in a $3 \mathrm{~mL}$ SPE cartridge. Optimization conditions were evaluated to determine the conditioning solvent, the loading solvent, washing, and the elution solvent that resulted in the highest DZM recovery.

2.8. Application of Polymer to Extract Diazepam from Spiked Serum and Selectivity Testing. The blood serum was obtained by centrifugation of the blood at a speed of $5000 \mathrm{rpm}$ for 5 minutes. Then, the upper part was taken. The blood serum was spiked with $2 \mathrm{ppm}$ diazepam in water. The spiked serum was passed through the MIP-SPE (molecular-imprinted solid-phase extraction) sorbent and the NIP-SPE (nonimprinted solid-phase extraction) sorbent. The SPE system used was the best condition determined in Section 2.7. The elution results were then analysed by HPLC using a mobile phase consisting of acetonitrile and water $(60: 40)$, with a flow rate of $1 \mathrm{~mL}$ per minute.

The serum was spiked with another drug that possibly exists in serum, besides diazepam. The optimum SPE system conditions were applied, and \% recovery was calculated for each compound.

2.9. Characterization of the Diazepam-Imprinted Polymer. Pore volume and surface area of the best polymer were analysed using a surface area analyser (SAA).

\section{Results and Discussion}

3.1. Computational Approach for the Prediction of MonomerTemplate Interactions. The interaction between the functional monomer (AAM and MMA) and the template (DZM) can be computationally predicted as a preliminary test. From Table 1, it can be seen that the monomer-template interaction between MMA and DZM gives a better binding affinity value $(-2.0 \mathrm{kcal} / \mathrm{mol})$ compared with the interaction between AAM and DZM $(-1.8 \mathrm{kcal} / \mathrm{mol})$. According to the literature, a smaller binding affinity value means greater interaction between molecules of a compound [23]. The interaction between MMA and DZM is the interaction of hydrogen bonds between carbonyl groups in the MMA and amine groups in DZM, whereas only hydrophobic interactions are formed between AAM and DZM.

3.2. Determination of the Association Constant of the Monomer-Template. Before the polymerization step, the association constant was evaluated to determine the ability of the MMA functional monomer in binding diazepam to form a stable complex in the prepolymerization solution with the titration method using UV-Vis spectrophotometry [24]. In Figure 1, it is seen that the association constant value is $1626 \mathrm{M}^{-1}$ for MMA and DZM and $200 \mathrm{M}^{-1}$ for ACM and DZM, each of which uses chloroform as the solvent. Chloroform was chosen because it is an aprotic nonpolar solvent, so it does not affect if hydrogen bond interactions are formed between templates and monomers [25]. The association test results using the UV titration method show that DZM as a template has more significant interactions with MMA than AAM. This also shows a correlation between the computational approach and UV-Vis titration. The association constant was calculated using the Benesi-Hildebrand equation, and a value of $1626 \mathrm{M}^{-1}$ for MMADZM was obtained (Figure 1). The higher the value of the association constant, the more stable the complex during polymerization and the better the imprinting effect $[23,24]$.

3.3. Synthesis of the Diazepam-Imprinted Polymer Using Bulk Polymerization. MMA was chosen as a functional monomer because it can form hydrogen bonds with the DZM template. MMA has oxygen atoms in carbonyl groups that can bind to the amine group in DZM, whereas AAM monomers were chosen because of the hydrophobic interactions that were determined to occur using the computation approach. Porogens used in the polymerization process must be able to dissolve all polymerization components. Chloroform was chosen because it can dissolve DZM and other components and it is aprotic, so it will not interfere with hydrogen bonds [25]. After the MIP is formed, the template must be removed from the polymer by extracting it. Extraction was carried out using methanol:acetic acid $(9: 1)$ continuously until all templates were eluted. 3 times of extraction are needed to complete the process. The purpose of the extraction was to remove DZM groups that were bound to the polymers and to form cavities that were a complement to DZM [26]. DZM is soluble in methanol, so it was used to extract the template. Acetic acid was added to disrupt the hydrogen bond between DZM and the functional monomer in order to facilitate the removal of DZM [27-29].

3.4. Evaluation of Binding Ability. To determine the binding ability and to find the optimum conditions for recognition of 
TABLE 1: Results from the computational approach used for the prediction of monomer-template interactions.

Template (diazepam)<smiles>CN1C(=O)CN=C(c2ccccc2)c2cc(Cl)ccc21</smiles><smiles>C=C(C)C(=O)OC</smiles>
Hydrogen bond, hydrophobic

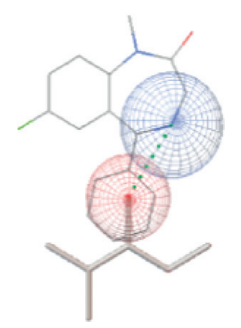

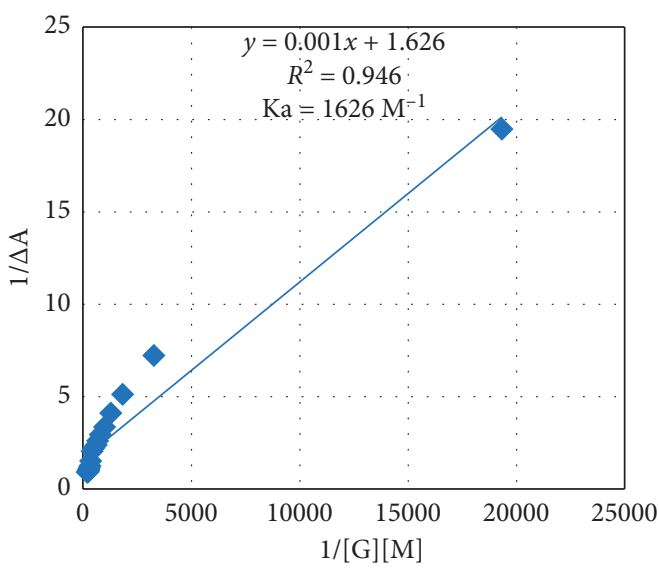

(a)

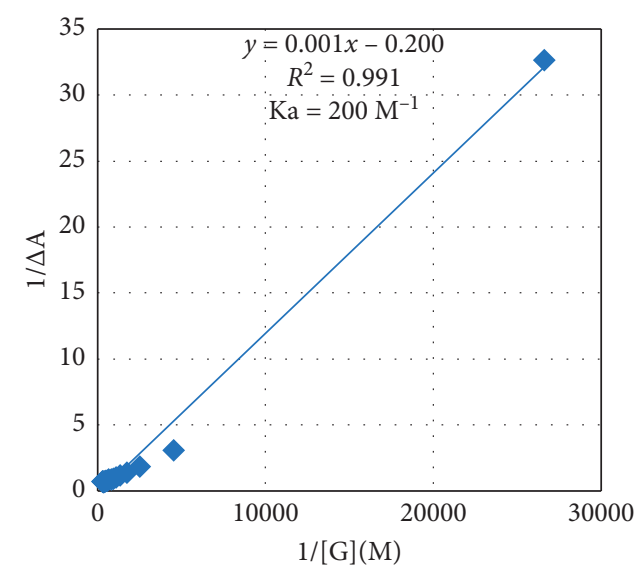

(b)

Figure 1: Association constant results for (a) MMA and DZM; (b) AAM and DZM.

the template by the prepared MIP, a standard solution of DZM (5 ppm) was initially prepared in various solvents, such as methanol, ethanol, and chloroform. The filtrate, which indicates the amount of unbound analytes, was measured. The DZM binding ability of the MIPs was investigated and compared to the binding to the NIP [20]. In Figure 2, it is known that the sorption ability of the MIP sorbent with the MMA monomer in chloroform gives the most excellent ability of $63.98 \pm 0.1 \%$ and NIP of $35.71 \pm 0.1 \%$, with an imprinting factor value of 1.7 , whereas in methanol solvents and ethanol it gave a consecutive value of, respectively, $63.436 \pm 0.04 \%$ for MIP and $34.58 \pm 0.1 \%$ for NIP, and $46.38 \pm 0.1 \%$ for MIP and $32.47 \% \pm 0.1 \%$ for NIP, with imprinting factor values of 1.8 and 1.4, respectively. In sorbents with AAM monomers, the value of the adsorption ability in chloroform was $43.62 \pm 0.04 \%$ and NIP was
$34.78 \pm 0.1 \%$, with an imprinting factor value of 1.25 , whereas in methanol and ethanol it gave a value of, respectively, $51.52 \pm 0.1 \%$ for MIP and $40.97 \pm 0.1 \%$ for NIP, and $18.80 \pm 0.1 \%$ for MIP and $17.52 \pm 0.1 \%$ for NIP with each imprinting factor value, respectively, at 1.26 and 1.07 . This is because chloroform is an aprotic nonpolar solvent, and the interaction between DZM and AAM monomers is hydrophobic, without hydrogen bonds, which is different from sorbents with MMA monomers, which form hydrogen bonds, thus providing greater adsorption ability. Solvents greatly affect the sorption ability of a sorbent, according to Tamayo et al. who stated that the optimum conditions for the template rebinding process involve use of the same solvent as that used during the synthesis of the MIPs [30].

The adsorption capacity of the MIP and NIP can be determined using the adsorption isotherm model. Isotherms 


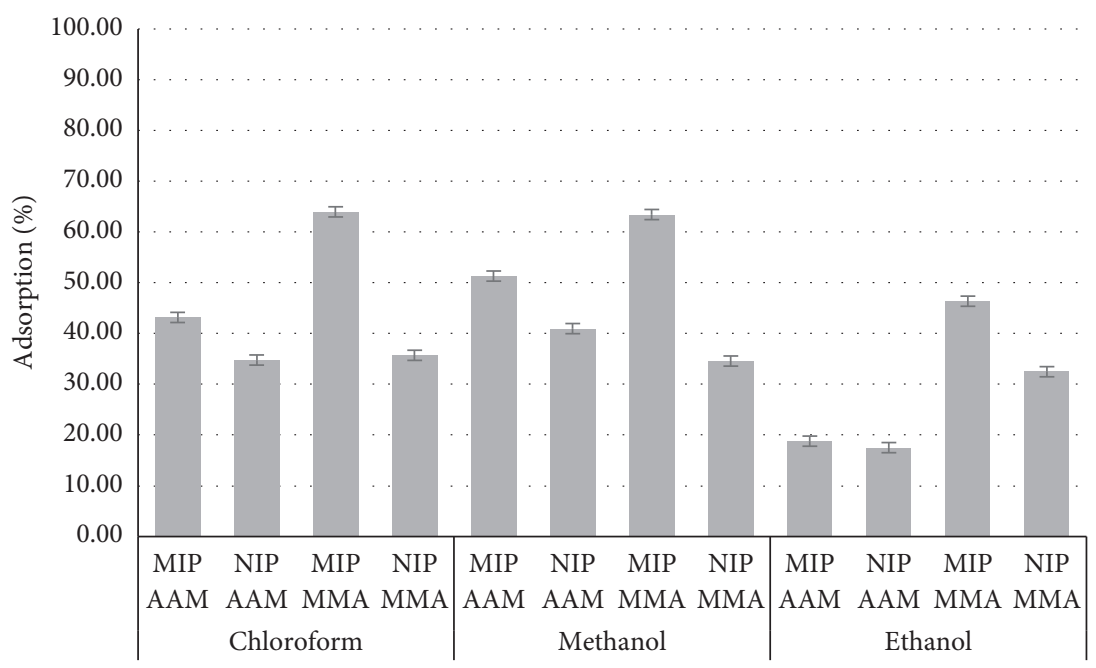

FIgURE 2: Adsorption percentage of MIP and NIP in chloroform, methanol, and ethanol. The error bars represent the standard deviation of three separate sample runs $(n=3)$.

can be matched using different models with different assumptions. The isotherm adsorption model that is suitable for use is the Freundlich isotherm model. This model is based on the assumption that sorbents have a nonhomogeneous surface, and each template molecule has different absorption potentials [31]. Based on Table 2, the MIP adsorption capacity with the functional monomer methyl methacrylate has a greater adsorption capacity value of $1.1607 \mathrm{mg} / \mathrm{g}$ for MIP and 0.0572 for NIP, while the adsorption capacity value for the sorbent with acrylamide monomers has a value of $0.1154 \mathrm{mg} / \mathrm{g}$ for MIP and $0.0401 \mathrm{mg} / \mathrm{g}$ for NIP.

3.5. Solid-Phase Extraction Optimization Conditions. The conditions of MI-SPE were obtained by conducting a series of solvent selection tests to be used at the conditioning, loading, washing, and eluting steps. The purpose of these steps was to determine the solvent that gives the highest recovery percentage during the elution step and a very small amount or almost zero percentage on another step. Optimization started with conditioning using $\mathrm{H}_{2} \mathrm{O}$ and acetonitrile $(9: 1)$ without flow rate adjustment, loading with diazepam solution in methanol, washing with acetone, and eluting with methanol and acetic acid (99:1), and using this condition, recovery percentage in the eluting step is below $10 \%$ for each polymer. We changed the optimization conditions by flow rate adjustment during the conditioning and loading steps with a flow rate $0.5 \mathrm{~mL} /$ minute. The other conditions were still the same. This condition resulted in a recovery percentage of $74.50 \pm 0.6 \%$ for MIP-MMA and $33.76 \pm 0.9 \%$ for NIP-MMA, while $50.74 \pm 0.9 \%$ for MIP-AAM and $29.99 \pm 1 \%$ for NIPAAM. According to that condition, we changed the washing solvent to a more nonpolar solvent, which was toluene. The other conditions were still the same. With this condition, we achieved $92.94 \pm 0.8 \%$ for MIP-MMA and NIP-MMA $62.04 \pm 0.9 \%$, while $50.27 \pm 0.8 \%$ for MIP-AAM and $30.92 \pm 0.9 \%$ for NIP-AAM. The result is shown in Figure 3 . Changing the washing solvent to a more nonpolar solvent proved that the interaction between DZM and the functional monomer consisted of hydrogen bonding, which would have been disrupted if we used a polar solvent, such as acetone, resulting in a lower recovery percentage. This result also confirm that the MIP has molecular recognition of imprinted cavities to DZM with hydrogen bonding interaction as the recovery is larger in more nonpolar solvent. Polymer swell in a good way can recognize DZM better in toluene. Based on the computational approach on prediction of monomer-template interaction, DZM has hydrogen bonding interaction with MMA and hydrophobic interaction with AAM. Recovery percentage of MIP-AAM was not changed after toluene was used as a washing solvent, and this confirms that the interaction is not hydrogen bonding. The optimal condition of SPE is then used for extracting DZM from a spiked serum sample.

3.6. Application of Polymer to Extract Diazepam from Spiked Serum and Selectivity Testing. As the result of the polymer made with MMA was better than AAM, we continued to apply MI-SPE and NI-SPE with the functional monomer MMA to separate DZM in serum samples. The accuracy of MI-SPE and NI-SPE was determined using the absolute method or stimulation (spiked-placebo recovery). The blood serum spiked with DZM, with a concentration of $5 \mathrm{mg} / \mathrm{L}$, and was then entered into the MI-SPE cartridge, which was made, and then extraction process was carried out as a condition of the optimization results and the percent recovery value was calculated. For comparison, the same procedure was carried out on NI-SPE, and testing was done three times so that the repeatability of each test could be observed. Figure 4 shows that application of serum sample separation using MI-SPE cartridges resulted in an average recovery percentage of $95.31 \pm 1.1 \%$, while NI-SPE gave an average recovery percentage of results of $60.83 \pm 0.3 \%$. Based on the results of the percent recovery rate, it is known that MI-SPE meets the requirements for recovering analytes from biological samples, which is more than $80 \%$ [32]. 
TABLE 2: Adsorption capacity of the MIP and NIP using the Freundlich isotherm adsorption.

\begin{tabular}{lcccc}
\hline Polymer & $\log K$ & $1 / n$ & $K(\mathrm{mg} / \mathrm{g})$ & $n$ \\
\hline MIP-MMA & -0.794 & 0.435 & 0.1607 & 2.2988 \\
NIP-MMA & -1.243 & 0.615 & 0.0572 & 1.6260 \\
MIP-ACM & -0.938 & 0.385 & 0.1154 & 2.5974 \\
NIP-ACM & -1.397 & 0.435 & 0.0401 & 2.2989 \\
\hline
\end{tabular}

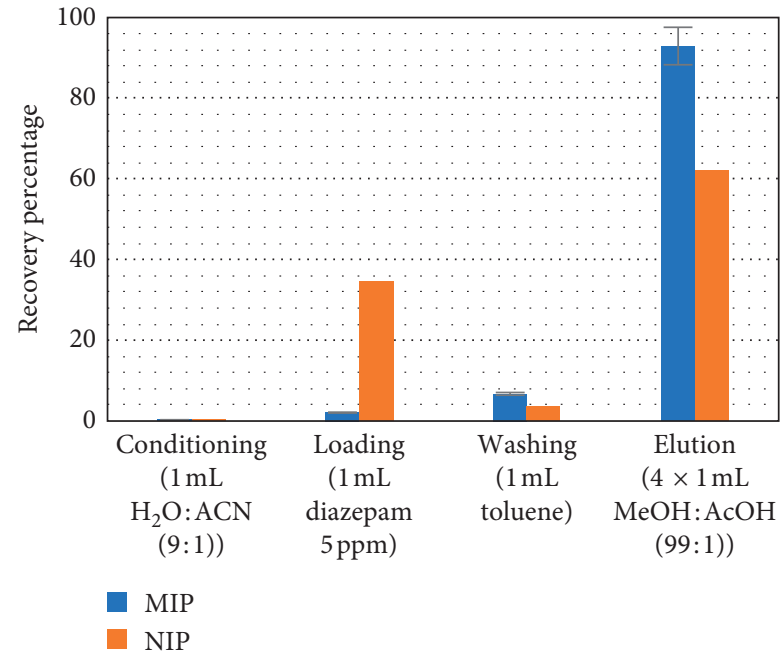

(a)

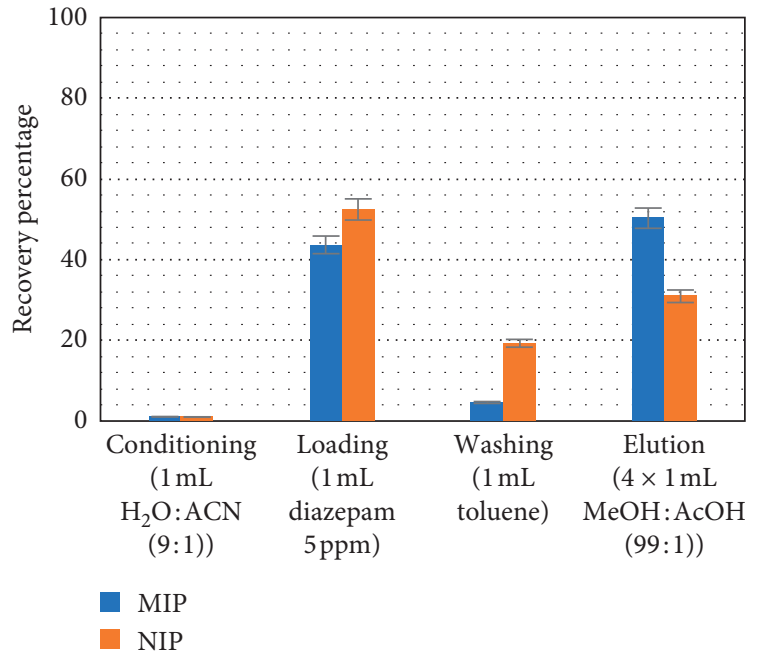

(b)

FIGURE 3: Recovery percentage of MMA polymer (a) and AAM polymer (b) with SPE condition (conditioning: $1 \mathrm{~mL} \mathrm{H}_{2} \mathrm{O}: \mathrm{ACN}(9: 1)$, loading: diazepam in $\mathrm{MeOH}$, washing: $1 \mathrm{~mL}$ toluene, eluting: $4 \times 1 \mathrm{~mL} \mathrm{MeOH}: \mathrm{AcOH}(99: 1))$. The error bars represent the standard deviation of three separate runs $(n=3)$.

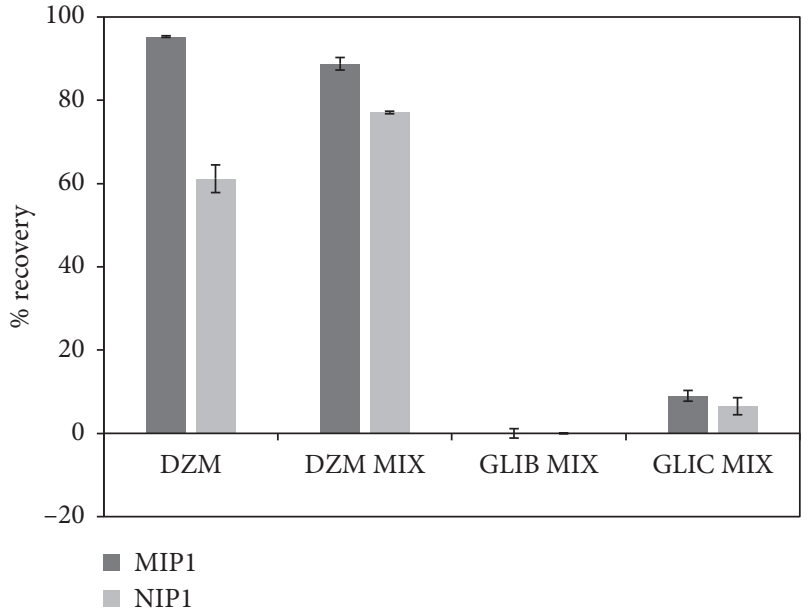

Figure 4: Percent recovery of DZM (alone and in a mixed solution of GLIB and GLIC). The error bars represent the standard deviation of three separate sample runs $(n=3)$.

Selectivity testing was carried out by adding other components to the serum besides DZM to see the effect of the presence of other compounds on the sorbent's ability to recognize DZM. Other compounds added to the serum were selected by glibenclamide (GLIB) and gliclazide (GLIC) because, based on testing using the HPLC conditions of
DZM, these two compounds can be analysed and responded to. In serum samples, DZM, GLIB, and GLIC were added with a concentration of $5 \mathrm{mg} / \mathrm{L}$ each. Figure 4 shows that the percent recovery of MI-SPE and NI-SPE to DZM was $88.78 \pm 2.0 \%$ and $77.07 \pm 0.2 \%$. GLIB was not detected in the elution section, and GLIC was $9.02 \pm 1.6 \%$ and $6.52 \pm 0.6 \%$. The results showed good selectivity of MI-SPE between MIP and NIP as there are differences in recovery percentage of DZM when it is alone and also in mixed with other drugs in serum. The chromatogram of the blood serum blank sample and spiked blood serum is shown in Figure 5 .

Comparison of the method result is given in Table 3. From the table, we could see that the method is comparable with recent research on DZM analysis in blood plasma [33] with SPE commercial being used as an extraction material and with MI-SPE made from methacrylic acid [17].

\subsection{Characterization of the Diazepam-Imprinted Polymer.} The physical characteristics of the polymer were determined using an SAA instrument (Quantachrome NovaWin) using the BET theory (Brunauer-Emmett-Teller) to determine the pore diameter, surface area, and pore volume of the polymer. Tests were carried out on MI-SPE and NI-SPE with the MMA monomer. The selection of the sorbent was carried 


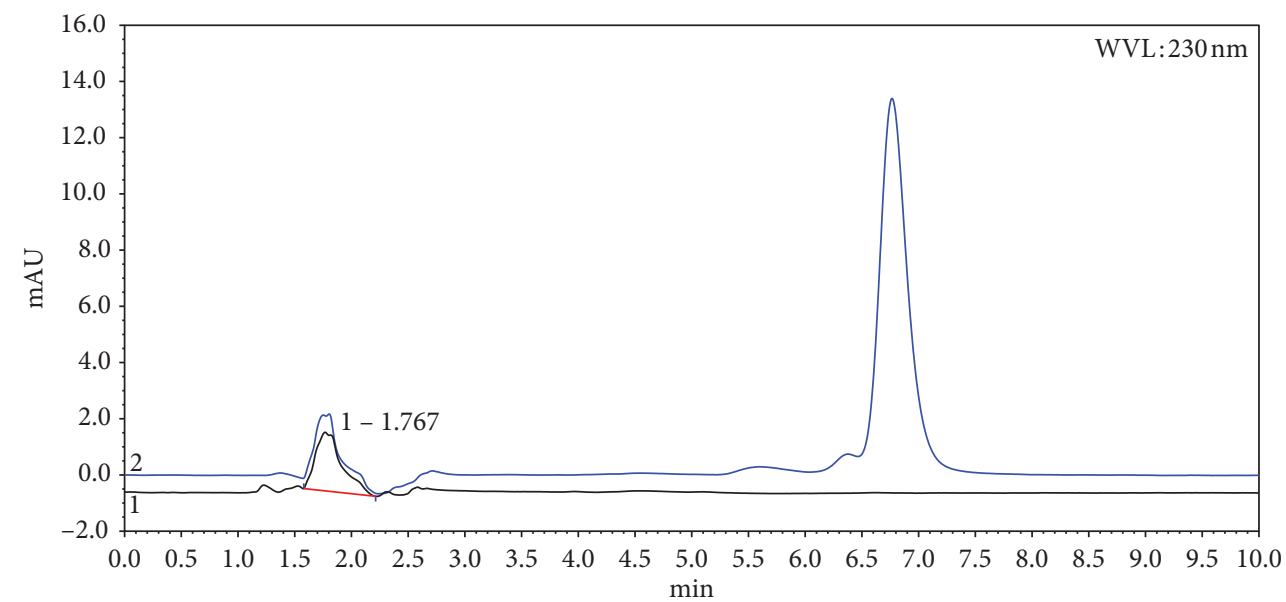

Figure 5: Chromatogram of blank sample (black) and blood serum spiked with mix compound and treated with MIP-MMA (blue).

TABLE 3: Comparison of repeatability, recovery, and LOQ values with other research studies.

\begin{tabular}{lcc}
\hline & Other research $[17,33]$ & Our results \\
\hline Repeatability (RSD) & $1.2 \%$ and $3 \%$ & $1.2 \%$ \\
Recovery & $88 \%$ and $97 \%$ & $95 \%$ \\
LOQ & $0.14 \mathrm{ng} / \mathrm{mL}$ and $2.5 \mathrm{ng} / \mathrm{mL}$ & $3.5 \mathrm{ng} / \mathrm{mL}$ \\
\hline
\end{tabular}

TABLE 4: Surface area analyser (SAA) results for MI-SPE (molecular-imprinted solid-phase extraction) MMA and NI-SPE (nonimprinted solid-phase extraction) MMA.

\begin{tabular}{lcc}
\hline Parameter & $\begin{array}{c}\text { MI-SPE } \\
\text { (MMA) }\end{array}$ & $\begin{array}{c}\text { NI-SPE } \\
\text { (MMA) }\end{array}$ \\
\hline Average pore diameter & $16.492 \mathrm{~nm}$ & $4.921 \mathrm{~nm}$ \\
\hline Adsorption pore size & & \\
distribution & & \\
$\quad$ Surface area & $133.822 \mathrm{mg}^{2} / \mathrm{g}$ & $23.051 \mathrm{~m}^{2} / \mathrm{g}$ \\
$\quad$ Pore volume & $0.295 \mathrm{~cm}^{3} / \mathrm{g}$ & $0.069 \mathrm{~cm}^{3} / \mathrm{g}$ \\
\hline Desorption pore size & & \\
distribution & & \\
$\quad$ Surface area & & \\
$\quad$ Pore volume & $84.126 \mathrm{mg}^{2} / \mathrm{g}$ & $16.609 \mathrm{~m}^{2} / \mathrm{g}$ \\
& $0.302 \mathrm{~cm}^{3} / \mathrm{g}$ & $0.041 \mathrm{~cm}^{3} / \mathrm{g}$ \\
\hline
\end{tabular}

out based on the best percent recovery value in testing the serum sample, which was $95.30 \pm 1.2 \%$ for MI-SPE and $61.1 \pm 0.3 \%$ for NI-SPE. Based on Table 4, the average pore diameter for MI-SPE is $16.449 \mathrm{~nm}$ and for NI-SPE it is $4.921 \mathrm{~nm}$. This shows that the type of pore is a mesopore type. According to the International Union of Pure and Applied Chemistry (IUPAC) classification, a sorbent has a mesoporous form if the pore diameter size is in the range of $2-50 \mathrm{~nm}$ or $20-500 \AA[34,35]$.

\section{Conclusions}

The MIP of DZM, with MMA as a functional monomer in chloroform, had better analytic performance than the MIP synthesized using AAM. Application of MI-SPE to spiked serum samples provided a good recovery percentage of $95.31 \pm 1.1 \%$ over $60.83 \pm 0.3 \%$ for the NIP alone, and it provided a lower recovery percentage when it was combined with another drug; however, it still passed official requirement for bioanalysis.

\section{Data Availability}

The data used to support the findings of this study are included in the article.

\section{Conflicts of Interest}

The authors declare that there are no conflicts of interest regarding the publication of this paper.

\section{Acknowledgments}

Funding from the Ministry of Research, Technology and Higher Education Republic of Indonesia, through Penelitian Dasar (PD) 2019 was greatly acknowledged.

\section{References}

[1] R. Kronstrand, I. Nystrom, M. Josefsson, and S. Hodgins, "Segmental ion spray LC-MS-MS analysis of benzodiazepines in hair of psychiatric patients," Journal of Analytical Toxicology, vol. 26, no. 7, pp. 479-484, 2002.

[2] Drug Enforcement Administration, Benzodiazepines, 2013.

[3] R. A. Anderson, M. M. Ariffin, P. A. G. Cormack, and E. I. Miller, "Comparison of molecularly imprinted solidphase extraction (MISPE) with classical solid-phase extraction (SPE) for the detection of benzodiazepines in post-mortem hair samples," Forensic Science International, vol. 174, no. 1, pp. 40-46, 2008.

[4] P. Cabarcos, M. J. Tabernero, I. Álvarez, P. López, P. Fernández, and A. M. Bermejo, "Analysis of six benzodiazepines in vitreous humor by high-performance liquid chromatography-photodiode-array detection," Journal of Analytical Toxicology, vol. 34, no. 9, pp. 539-542, 2010.

[5] A. Pyka, E. Wiatr, K. Kwiska, and D. Gurak, "Validation thin layer chromatography for the determination of naproxen in tablets and comparison with a pharmacopeil method," Journal of Liquid Chromatography \& Related Technologies, vol. 34, no. 10-11, pp. 829-847, 2011. 
[6] M. Bakavoli and M. Kaykhaii, "Quantitative determination of diazepam, nitrazepam and flunitrazepam in tablets using thin-layer chromatography-densitometry technique," Journal of Pharmaceutical and Biomedical Analysis, vol. 31, no. 6, pp. 1185-1189, 2003.

[7] H. H. Lee, J. F. Lee, S. Y. Lin et al., "Simultaneous quantification of urine flunitrazepam, nimetazepam and nitrazepam by using liquid chromatography tandem mass spectrometry," Clinica Chimica Acta, vol. 420, pp. 134-139, 2013.

[8] L. M. L. Carvalho, A. X Linhares, and J. R. Trigo, "Determination of drug levels and the effect of diazepam on the growth of necrophagous flies of forensic importance in southeastern Brazil," Forensic Science International, vol. 120, no. 1-2, pp. 140-144, 2001.

[9] K. Tyszczuk, "Determination of diazepam, temazepam and oxazepam at the lead film electrode by adsorptive cathodic stripping voltammetry," Electroanalysis, vol. 22, no. 17-18, pp. 1975-1984, 2010.

[10] F. Ahmadi and E. Karamian, "Computational aided-molecular imprinted polymer design for solid phase extraction of metaproterenol from plasma and determination by voltammetry using modified carbon nanotube electrode," Iranian Journal of Pharmaceutical Research, vol. 13, no. 2, pp. 417430, 2014.

[11] S. Lay, H.-n. Yu, B.-x. Hu, and S.-r. Shen, "Molecularly imprinted polymers as the extracted sorbents of clenbuterol ahead of liquid chromatographic determination," Journal of Zhejiang University-Science B, vol. 17, no. 6, pp. 465-475, 2016.

[12] A. N. Hasanah, D. Rahayu, R. Pratiwi et al., "Extraction of atenolol from spiked blood serum using a molecularly imprinted polymer sorbent obtained by precipitation polymerization," Heliyon, vol. 5, no. 4, 2019.

[13] R.G. Machicote, M. A. Castillo, M.E. Pacheco, and L. Bruzzone, "A molecular imprinted polymer as a flow-trough optical sensor for oxazepam," Journal of Analytical Methods in Chemistry, vol. 2018, Article ID 6302609, 7 pages, 2018.

[14] X.-H. Wang, L.-F. Xie, Q. Dong, H.-L. Liu, Y.-P. Huang, and Z.-S. Liu, "Synthesis of monodisperse molecularly imprinted microspheres with multi-recognition ability via precipitation polymerization for the selective extraction of cyromazine, melamine, triamterene and trimethoprim," Journal of Chromatography B, vol. 1007, pp. 127-131, 2015.

[15] E. C. Figueiredo, R. Sparrapan, G. B. Sanvido, M. G. Santos, M. A. Z. Arruda, and M. N. Eberlin, "Quantitation of drugs via molecularly imprinted polymer solid phase extraction and electrospray ionization mass spectrometry: benzodiazepines in human plasma," The Analyst, vol. 136, no. 18, p. 3753, 2011.

[16] Y. Liu, K. Hoshina, and J. Haginaka, "Monodispersed, molecularly imprinted polymers for cinchonidine by precipitation polymerization," Talanta, vol. 80, no. 5, pp. 1713-1718, 2010.

[17] Q. Su, C. Zeng, Y. Tang, D. E. Finlow, and M. Cao, "Evaluation of diazepam-molecularly imprinted microspheres for the separation of diazepam and its main metabolite from body fluid samples," Journal of Chromatographic Science, vol. 50, no. 7, pp. 608-614, 2012.

[18] S. Song, A. Wu, X. Shi, R. Li, Z. Lin, and D. Zhang, "Development and application of molecularly imprinted polymers as solid-phase sorbents for erythromycin extraction," Analytical and Bioanalytical Chemistry, vol. 390, no. 8, pp. 2141-2150, 2008.

[19] P. Thordarson, "Determining association constants from titration experiments in supramolecular Chemistry," Chemical Society Reviews, vol. 40, no. 3, pp. 1305-1323, 2011.
[20] H. Yan and K. Row, "Characteristic and synthetic approach of molecularly imprinted polymer," International Journal of Molecular Sciences, vol. 7, no. 5, pp. 155-178, 2006.

[21] A. N. Hasanah, R. E. Kartasasmi, and S. Ibrahim, "Synthesis and application of glibenclamide imprinted polymer for solid phase extraction in serum samples using itaconic acid as functional monomer," Journal of Applied Sciences, vol. 15, no. 11, pp. 1288-1296, 2015.

[22] S. Ansari and A. Ghorbani, "Molecularly imprinted polymers (MIP) for selective solid phase extraction of celecoxib in urine sampeles followed by high performance liquid chromatography," Journal of Chemical Health Risks, vol. 7, 2017.

[23] M. Yan, "The covalent and other stoichiometric approaches," Molecularly Imprinted Materials: Science and Technology, pp. 59-92, CRC Press, Boca Raton, FL, USA, 2005.

[24] S. Scorrano, L. Mergola, R. Del Sole, and G. Vasapollo, "Synthesis of molecularly imprinted polymers for amino acid derivates by using different functional monomers," International Journal of Molecular Sciences, vol. 12, no. 3, pp. 1735-1743, 2011.

[25] J. O’Mahony, A. Molinelli, K. Nolan, M. R. Smyth, and B. Mizaikoff, "Towards the rational development of molecularly imprinted polymers: $1 \mathrm{H}$ NMR studies on hydrophobicity and ion-pair interactions as driving forces for selectivity," Biosensors and Bioelectronics, vol. 20, no. 9, pp. 884-1893, 2005.

[26] S. N. N. S. Hashim, R. I. Boysen, L. J. Schwarz, B. Danylec, and M. T. W. Hearn, "A comparison of covalent and non-covalent imprinting strategies for the synthesis of stigmasterol imprinted polymers," Journal of Chromatography A, vol. 1359, pp. 35-43, 2014.

[27] K. F. Lim and C. I. Holdsworth, "Effect of formulation on the binding efficiency and selectivity of precipitation molecularly imprinted polymers," Molecules, vol. 23, no. 11, p. 2996, 2018.

[28] L. Chen, X. Wang, W. Lu, X. Wu, and J. Li, "Molecular imprinting: perspectives and applications," Chemical Society Reviews, vol. 45, pp. 1-75, 2016.

[29] A. N. Hasanah, T. N. Sari, N. Wijaya, R. E. Kartasasmita, and S. Ibrahim, "Study of the binding ability of molecular imprinted solid phase extraction for glibenclamide by optimizing template: monomer: crosslinker ratio," International Journal of Chemical Science, vol. 12, no. 3, pp. 1288-1296, 2014.

[30] F. G. Tamayo, J. L. Casillas, and A. Martin-Esteban, "Clean up of phenylurea herbicides in plant sample extracts using molecularly imprinted polymers," Analytical and Bioanalytical Chemistry, vol. 381, no. 6, pp. 1234-1240, 2005.

[31] P. Kadhirvel, Molecular imprints for pharmaceutical drugs of different classes: comparison of sol-gel and acrylic approaches in different formats, PhD. thesis, Universidado the Porto, Porto, Portugal, 2013.

[32] FDA, Guidance for Industry: Bioanalytical Method Validation, U.S. Department of Health and Human Services, Washington, DC, USA, 2013.

[33] L. Mercolini, R. Mandrioli, C. Lannelo, F. Matrisciano, N. Ferdinando, and M. A. Raggi, "Simultaneous analysis of diazepam and its metabolites in rat -plasma and brain tissue by HPLC-UV and SPE," Talanta, vol. 80, pp. 229-285, 2009.

[34] Y. Cai, Y. Chen, X. Hong, Z. Liu, and W. Yuan, "Porous microsphere and its applications," International Journal of Nanomedicine, vol. 8, pp. 1111-1120, 2013.

[35] X. Y. Zhao, J. P. Tu, Y. Lu et al., "Graphene-coated mesoporous carbon/sulfur cathode with enhanced cycling stability," Electrochimica Acta, vol. 113, pp. 256-262, 2013. 